\title{
Evaluación de la aceptabilidad del pan reducido en sodio en consumidores de la Región Metropolitana de Chile
}

\author{
Acceptability assessment of bread \\ reduced sodium in the \\ Metropolitan Region of Chile
}

\begin{abstract}
Introduction: Due to the disturbing increase in cardiovascular disease, in part associated with the consumption of salt, a pilot program was initiated in 2010 between the Ministry of Health, the Federation of Industrial Bakers and the Chilean Association of supermarkets to gradually reduce the amount of salt in bread. Aim: to evaluate the overall acceptability of salt-reduced bread in a pilot program compared with unmodified bread in bakeries of Santiago. Methodology: A cross-sectional analytical study was performed. Random selection of 5 intervention bakeries and 5 control bakeries. A survey of 175 consumers per group was carried out in order to assess the acceptability of bread in relation to 5 sensory parameters, with a hedonic scale of 5 points. An index of overall acceptability (5-25 points) was developed qualifying as good acceptability a value $\geq 22$ points. Results: Good acceptability was found in all parameters studied. A statistically significant difference was found for the control group in the rating of the flavor $(p=0,01)$ and global acceptability of bread ( $p=0$, 02). Multivariate analysis showed better acceptability in persons over 60 years of age $(p=0,02)$ and in control bakeries $(p=0,01)$. Conclusions: Better acceptability was found in some parameters in the control group bread, although the sodium concentration is not the only factor to explain the acceptability.

Key words: sodium, bread, acceptability, arterial hypertension, Chile.
\end{abstract}

\section{INTRODUCCIÓN}

Ante el preocupante aumento de las enfermedades cardiovasculares en el mundo, en parte asociadas al consumo excesivo de sodio $(1,2)$, varios países se han propuesto aunar fuerzas y desarrollado estrategias globales para este fin (3-8).

En la actualidad, tanto la Organización Mundial de la Salud como la Organización Panamericana de la Salud se encuentran desarrollando grupos de trabajos a nivel internacional con expertos de distintas naciones para generar recomendaciones a fin de cumplir la meta de reducir el nivel mundial del consumo de sodio por persona a menos de $2.000 \mathrm{mg}$ sodio/ día (5 g sal /día ) (9).

En nuestro país existe un consumo promedio de pan del orden de $250 \mathrm{~g} /$ persona/día, con una concentración de sodio cercana a $800 \mathrm{mg} / 100 \mathrm{~g}$, lo que determina un consumo promedio de sodio de aproximadamente $2 \mathrm{~g} /$ día, sólo por esta vía.
Karen Valenzuela L. (1) Vilma Quitral R. (2) Fernando Zavala M. (3) Beatriz Villanueva A. (4) Eduardo Atalah S. (2)

1) Servicio Medicina, Hospital de Carabineros. Santiago, Chile (2) Departamento de Nutrición, Facultad de Medicina, Universidad de Chile. Santiago, Chile. (3) Alumno Facultad de Medicina, Universidad Andrés Bello. Santiago, Chile. (4) Vitaclinic. Santiago, Chile.

Dirigir la correspondencia a: Karen Valenzuela $L$. Servicio Medicina, Hospital de Carabineros Simón Bolívar \# 2200, Nuñoa. Santiago, Chile Teléfono: 022 9278347 Email: drakarenvalenzuela@gmail.com

Este trabajo fue recibido el 22 de Octubre de 2013 y aceptado para ser publicado el 15 de Enero de 2014.
En total se estima un consumo promedio de sodio de 4.800 $\mathrm{mg} /$ día por persona (12 g sal por persona/día), más del doble de las recomendaciones internacionales (10).

Para disminuir la ingesta de sal en la población, Chile se ha sumado a otras iniciativas mundiales usando como eje de su campaña la disminución progresiva de sal en el pan. De esta manera en Septiembre del año 2010 el MINSAL acordó con la FECHIPAN y la ASACH disminuir en forma gradual el contenido de sal en la elaboración del pan tipo hallulla y marraqueta. Se estableció un programa piloto en alrededor de 100 panaderías de Santiago y Regiones con el objetivo de disminuir al 2011 en 25\% la sal agregada al pan, y en $50 \%$ para el año 2014, para llegar a una cifra de sodio de 400 $\mathrm{mg} / 100 \mathrm{~g}$ de pan (11).

La elaboración de un pan con menos sal representa un desafío para la industria, ya que la sal cumple importantes 
funciones en su elaboración, mejorando sus características organolépticas, reológicas y de estabilidad (12). La disminución de la sal puede determinar menor aceptabilidad por parte de los consumidores, incluso puede presentarse agrio, ácido, con sabor a levadura, más añejo y seco, de acuerdo a descripciones dadas en análisis sensorial de pan reducido en sal $(13,14)$.

Considerando los importantes efectos que puede determinar desde el punto de vista sanitario y para la industria del rubro el modificar un producto tradicional de alto consumo, se hace necesario conocer el grado de aceptabilidad de los consumidores frente a este pan con menor contenido de sal.

El objetivo principal de este estudio fue evaluar la aceptabilidad general del pan tipo marraqueta y hallulla reducido en un $25 \%$ de sodio del programa piloto MINSAL-FECHIPANASACH en comparación con un pan control, en panaderías de Santiago.

\section{MATERIALES Y MÉTODOS}

Diseño: Estudio tipo transversal analítico.

Universo: 95 panaderías participantes del programa piloto MINSAL-FECHIPAN-ASACH ubicadas en diferentes comunas del Gran Santiago y las panaderías no adheridas al programa.

Muestra: Clientes de 5 panaderías participantes del programa piloto (grupo intervenido) y 5 panaderías no participantes (grupo control) de las mismas comunas anteriores, que fueron seleccionadas en forma aleatoria simple.

Tamaño de muestra: Como no existe información nacional sobre el posible efecto de la reducción de la sal en la aceptabilidad del pan, se estimó arbitrariamente un 95\% de aceptabilidad del pan tradicional, un $10 \%$ menos en el programa piloto, un nivel de significancia $\alpha<0,05$, poder del test de $90 \%$ y un test de una cola. Ello determinó la necesidad de realizar 153 encuestas en cada grupo y asumiendo un $10 \%$ de respuestas incompletas, se amplió la muestra a 175 encuestas, realizándose 35 encuestas en cada una de las 10 panaderías seleccionadas.

Criterios de inclusión: Compradores de 18 a 70 años, haber comprado más de alguna vez en el local encuestado, no presentar alteraciones cognitivas ni sensoriales evidentes o referidas por el consumidor al momento de la entrevista y haber comprado pan marraqueta y/o hallulla.

Instrumento: Para medir la aceptabilidad del pan se aplicó una encuesta a los consumidores de ambos grupos durante el primer semestre del año 2012. Antes de su empleo, la encuesta fue aplicada en 20 personas con características similares a la población a estudiar y en función de los resultados obtenidos se reformularon algunas preguntas que no fueron adecuadamente comprendidas.

Se analizó la opinión del consumidor respecto al color, olor, sabor, textura y calificación general del pan de ese establecimiento, expresadas en una escala hedónica de 5 puntos: 1= Me disgusta mucho; $2=$ Me disgusta; $3=$ No me gusta ni me disgusta; 4= Me gusta; $5=$ Me gusta mucho. La aceptabilidad global del pan fue calculada a partir de la suma de las 5 variables anteriores, con escala de 5 a 25 . Se consideró buena aceptabilidad un puntaje $\geq$ a 22 puntos.

La encuesta incluyó además información sobre el sexo, edad, escolaridad, interés por consumir pan reducido en sal y el conocimiento sobre posibles efectos negativos asociados al consumo de sal.

Análisis estadístico. Los datos de las encuestas fueron digitados y verificados en una planilla Excel y analizados con el programa STATA 11.1. Se analizó la normalidad de las va- riables y las variables sin distribución normal se expresaron en medianas y percentiles como medidas de tendencia central. En las variables nominales y categóricas, se analizó la distribución de frecuencia y se aplicó el test de $x^{2}$ para la comparación entre los grupos.

Mediante regresiones logísticas se realizaron análisis univariados de la relación entre las variables estudiadas (sexo, escolaridad, edad, tipo de panadería) con una buena aceptabilidad general del pan (puntaje $\geq 22$ ). Con las variables que resultaron significativas se realizó un análisis multivariado. En todos los análisis estadísticos se consideró significativo un valor de $p<0,05$.

Aspectos éticos: El presente estudio fue aprobado por el Comité de Ética de la Facultad de Medicina, Universidad de Chile.

\section{RESULTADOS}

A fines del año 2011 y principios del año 2014 se entrevistaron 236 personas en el grupo control y 213 en el grupo intervenido, con $25,8 \%$ y $17,8 \%$ de rechazo en cada grupo, respectivamente. La principal causa de rechazo fue la falta de tiempo ( $77 \%$ del total). En $2 \%$ de las encuestas se registraron datos incompletos.

Un $61,4 \%$ de los encuestados era de sexo femenino. La mediana de edad de los consumidores de ambos grupos fue 45 años (p 25-75, 37-55 años), sin diferencias significativas en función del sexo o tipo de panaderías. Un 94,8\% de la población declaró estar en conocimiento sobre los efectos nocivos de la sal/sodio en el organismo, con un porcentaje algo mayor en el grupo control $(97,7 \%$ vs $91,9 \% p=0,04)$.

El $60,6 \%$ de los consumidores encuestados estaba interesado en consumir un pan con menos sal/sodio, aunque al $40 \%$ restante no les interesaba o le era indiferente, sin diferencias significativas por grupo de estudio. Al realizar el mismo análisis según edad se observó un mayor interés en consumir menos sal en las personas de 60 años o más $(p=0,003)$.

La distribución del puntaje asignado en cada ítem evaluado se muestra en la tabla 1. Considerando la baja proporción de respuestas en las categorías 1, 2 y 3 se agruparon estas tres categorías, lo que podría considerarse como aceptabilidad regular. En general predomina la respuesta "me gusta" en todos los ítems, aunque también hay una alta proporción de respuestas "me gusta mucho". El comportamiento fue algo más favorable en sabor $(p=0,01)$ y color $(p=0,05)$ en el grupo no intervenido, sin diferencias en la calificación del olor, textura, ni calificación general del pan. La mediana de puntaje en todos los ítems sensoriales fue de 4 puntos ( $p 25-75,4-5$ ), en ambos grupos, lo que refleja una buena aceptabilidad del pan (datos no mostrados).

En la aceptabilidad global del pan, que incluye los cinco ítems anteriores, se observó una diferencia estadísticamente significativa a favor de grupo control $(p=0,02)$, con una diferencia de 14,7 puntos porcentuales en la categoría de mayor puntuación (tabla 2).

Al realizar un análisis de regresión logística univariada de la influencia de las variables de control (sexo, edad y escolaridad) sobre la buena la aceptabilidad global del pan (calificación $\geq 22$ puntos) se observó que sólo la edad de los consumidores influyó en forma significativa con una mayor aceptabilidad en el grupo de personas mayores de 60 años $(p=0,02$ OR=1,41 IC $95 \%=[1,04-1,92])$. El mismo análisis sobre la influencia del tipo de panadería mostró mejores resultados a favor de las panaderías controles $(p=0,01$ OR=1,76 IC 95\%= [1,15-2,72]).

Un análisis multivariado de los factores que se asociaron 
con la aceptabilidad global del pan, mostró mejores resultados en las panaderías control (OR 1,75 IC= 1,13 - 2,71) y en personas de mayor edad, aunque explican una baja proporción de las diferencias observadas (pseudo $R^{2}=0,02$ ) (tabla 3 ).

\section{DISCUSIÓN}

Si bien el número inicial de personas a entrevistar por grupo se fijó en 175 consumidores, esta cifra debió aumentarse debido al rechazo a participar por parte de los clientes, situación esperable por la ya conocida dificultad al trabajar con encuestas. El principal motivo de rechazo fue la falta de tiempo, situación que no es de extrañar debido al ritmo de vida actual y a la ubicación en lugares "de paso" de varias de las panaderías. Lamentablemente no existe información sobre las características del grupo que no aceptó participar en relación al grupo encuestado, para evaluar posibles sesgos en la muestra, lo que podría limitar la representatividad del estudio.

Llama la atención que cerca del $40 \%$ de la población no estaba interesada o les era indiferente consumir pan con menor concentración de sal, a pesar de que la mayoría declaraba estar al tanto de que la sal es nociva para la salud. Dicha situación sin duda limita la posibilidad de obtener buenos resultados en campañas de salud que requieran participación activa de la población. Aunque el grupo control presentó mayor interés por consumir pan con menos sal, esto podría deberse al azar, ya que los encuestados no tenían información sobre las características del pan que estaban comprando.

Las personas mayores de 60 años presentaron un mayor interés en consumir un pan con menos sodio, lo cual podría explicarse por presentar mayor número de patologías crónicas cardiovasculares o estar en tratamiento médico, por lo que estarían más dispuestos a cuidar su salud y preferir alimentos más saludables.

Al evaluar las características organolépticas del pan, se observaron diferencias significativas a favor de grupo control sólo en sabor con un aumento de $12 \%$ en la puntuación máxima. La información aportada por estudios internacionales no es concluyente sobre la influencia del sodio en el sabor del pan, ni sobre cuánto se puede disminuir su cantidad sin afectar el sabor. Algunos autores muestran que se pueden realizar reducciones de 10 y $20 \%$ sin alterar el sabor del pan $(15,16)$. Una revisión reciente señala que para algunos investigadores el sodio contenido en el pan puede reducirse entre 25 y $50 \%$

TABLA 1

Distribución de la calificación obtenida para los diferentes atributos sensoriales del pan según grupo estudiado.

\begin{tabular}{|c|c|c|c|c|c|c|}
\hline \multirow[t]{2}{*}{ Variable } & \multicolumn{2}{|c|}{ Grupo intervenido } & \multicolumn{2}{|c|}{ Grupo control } & \multicolumn{2}{|c|}{ Total } \\
\hline & $\mathrm{n}$ & $\%$ & $\mathrm{n}$ & $\%$ & $\mathrm{n}$ & $\%$ \\
\hline \multicolumn{7}{|l|}{ Sabor } \\
\hline $1-3$ & 24 & 13,7 & 29 & 16,6 & 53 & 15,1 \\
\hline 4 & 95 & 54,3 & 69 & 39,4 & 164 & 46,9 \\
\hline \multirow[t]{2}{*}{5} & 56 & 32,0 & 77 & 44,0 & 133 & 38,0 \\
\hline & & & & & $x^{2} 7,9$ & $p=0,01$ \\
\hline \multicolumn{7}{|l|}{ Olor } \\
\hline $1-3$ & 31 & 17,7 & 33 & 18,8 & 64 & 18,3 \\
\hline 4 & 77 & 44,0 & 67 & 38,3 & 144 & 41,1 \\
\hline \multirow[t]{2}{*}{5} & 67 & 38,3 & 75 & 42,9 & 142 & 40,6 \\
\hline & & & & & $x^{2} 1,2$ & $p=0,5$ \\
\hline \multicolumn{7}{|l|}{ Color } \\
\hline $1-3$ & 43 & 24,6 & 29 & 16,6 & 72 & 20,6 \\
\hline 4 & 86 & 49,1 & 82 & 46,9 & 168 & 48,0 \\
\hline \multirow[t]{2}{*}{5} & 46 & 26,3 & 64 & 36,5 & 110 & 31,4 \\
\hline & & & & & $x^{2} 5,7$ & $p=0,05$ \\
\hline \multicolumn{7}{|l|}{ Textura } \\
\hline $1-3$ & 31 & 17,7 & 19 & 0,9 & 50 & 14,3 \\
\hline 4 & 74 & 42,3 & 72 & 41,1 & 146 & 41,7 \\
\hline \multirow[t]{2}{*}{5} & 70 & 40,0 & 84 & 48,0 & 154 & 44,0 \\
\hline & & & & & $x^{2} 4,1$ & $p=0,12$ \\
\hline \multicolumn{7}{|c|}{ Calificación general } \\
\hline $1-3$ & 31 & 17,7 & 23 & 13,2 & 54 & 15,4 \\
\hline 4 & 88 & 50,3 & 90 & 51,4 & 178 & 50,9 \\
\hline \multirow[t]{2}{*}{5} & 56 & 32,0 & 62 & 35,4 & 118 & 33,7 \\
\hline & & & & & $\times 21,5$ & $p=0,4$ \\
\hline Total & 175 & 100,0 & 175 & 100,0 & 350 & 100,0 \\
\hline
\end{tabular}


sin afectar el sabor, mientras que para otros preparar un pan con un 50\% menos de sodio sería suficiente para alterar el sabor de éste (17). En un estudio conducido por Girgis et al., en que se comparó sucesivamente pan blanco estándar v/s pan blanco preparado con reducción de un 5\% de sodio semanalmente por 6 semanas, se obtuvo como resultado que esta disminución paulatina no afecta la intensidad del sabor del pan (18). Mientras que Bolhuis et al. al realizar la evaluación con pan integral, observaron que una reducción de hasta un $52 \%$ de sal no afectó el consumo respecto al pan control (sin reducción de sal) (19).

En nuestro país, en una encuesta mencionada por INDUPAN, se indica que un $67 \%$ de los encuestados prefiere un pan con menos sal (aunque no se indica la concentración de sal) versus un pan normal, mientras que un $8 \%$ no nota la diferencia entre ambos tipos de pan (20). A la fecha, en consecuencia, aun no se encuentra establecido un "límite de seguridad" para no afectar la aceptabilidad del pan.

En este estudio se obtuvieron diferencias significativas en la aceptabilidad global del pan, pero no así en la calificación general. Ello podría explicarse por el hecho que la calificación general es una puntuación dada por el propio consumidor, en tanto la aceptabilidad global es un resumen de los 5 ítems estudiados, dentro los cuales se encuentra la calificación general. De esta forma la aceptabilidad global refleja un puntaje que abarca las distintas características organolépticas del pan siendo una variable más completa que aporta más información. Teniendo en cuenta lo anterior, el pan del grupo control es globalmente mejor evaluado que el pan del grupo intervenido. Sin embargo en un estudio paralelo realizado en el mismo grupo de panes analizados en la presente investigación se demostró que tanto los controles como los intervenidos presentaron concentraciones de sodio cercanas a los $600 \mathrm{mg} / 100$ $\mathrm{g}$ de pan, sin diferencias significativas entre ellos, por lo cual el grupo control no estaría actuando realmente como tal (21). De esta manera, tal diferencia en la aceptabilidad no necesariamente estaría explicada por la concentración de sodio en las muestras evaluadas, sino más bien por la panadería que lo elabora. Según el análisis multivariado el modelo explica una baja proporción de la aceptabilidad (pseudo R2 2\%), lo que indica que muchas otras variables pueden estar influyendo sobre una mejor aceptación del pan. Dentro de ellas se pueden mencionar distintas recetas para elaborar el pan, tiempos de horneado, tipo y tiempo de amasado, entre otras.

Dentro de las limitaciones de este estudio, se reconoce que el número de personas encuestadas y su distribución probablemente no fue suficientemente adecuada para obtener una muestra más representativa del universo en estudio.

Otro hecho importante a considerar es la dificultad para aplicar encuestas en locales comerciales, punto importante a considerar para investigaciones futuras. En este estudio hubo cierta reticencia por parte los locales a dar autorización para ser encuestados. Mencionar la palabra "evaluación" dentro de los objetivos del estudio al parecer fue la principal piedra de tope para que las autorizaciones se consiguieran con mayor fluidez.

En nuestro país no se han publicado estudios previos sobre la aceptabilidad del pan en los consumidores y esta investigación puede representar un primer paso para análisis futuros en este tema. Así mismo pretende servir como referencia para quienes trabajen en la difícil tarea de lograr reducir el consumo de sal/sodio en la población sin afectar la calidad de los productos a utilizar.

Se puede concluir que el pan del grupo control presenta mejor aceptabilidad global, aunque no sería este elemento el único factor que estaría influyendo en la aceptabilidad de este alimento por parte de los consumidores. Se necesitan estudios más extensos para conocer una realidad más representativa a nivel nacional.

\section{TABLA 2}

Distribución de la calificación obtenida en aceptabilidad global según la suma de los atributos sensoriales del pan, por grupo.

\begin{tabular}{|c|c|c|c|c|c|c|}
\hline \multirow{2}{*}{$\begin{array}{l}\text { Puntaje Global } \\
12-19\end{array}$} & \multicolumn{2}{|c|}{$\begin{array}{c}\text { Grupo intervenido } \\
\text { Número } \%\end{array}$} & \multicolumn{2}{|c|}{$\begin{array}{l}\text { Grupo control } \\
\text { Número \% }\end{array}$} & \multicolumn{2}{|c|}{$\begin{array}{c}\text { Total } \\
\text { Número \% }\end{array}$} \\
\hline & 56 & 32,8 & 42 & 24,0 & 98 & 28,2 \\
\hline $20-21$ & 59 & 3,9 & 49 & 28,0 & 108 & 31,0 \\
\hline $22-25$ & 60 & 33,3 & 84 & 48,0 & 144 & 40,8 \\
\hline Total & 175 & 100,0 & 175 & 100,0 & 350 & 100,0 \\
\hline Test $\mathrm{Chi}^{2}$ & & & & & $x^{2} 7,8$ & $p=0,02$ \\
\hline
\end{tabular}

\section{TABLA 3}

Regresión logística multivariada para analizar una mayor aceptabilidad global del pan.

\begin{tabular}{lccc}
\hline Aceptabilidad global & OR & I. Confianza & (95\%) \\
& & $1,13-2,71$ & 0,011 \\
Panadería control & 1,75 & $1,04-1,91$ & 0,026 \\
Edad > 60 años & 1,41 & & 0,02 \\
Pseudo R & & & \\
\hline
\end{tabular}




\section{RESUMEN}

Introducción: Ante el aumento de enfermedades cardiovasculares en Chile, asociadas al alto consumo de sodio, el año 2010 se inició un programa piloto entre el Ministerio de Salud, la Federación de Industriales Panaderos y la Asociación Chilena de Supermercados para disminuir gradualmente la concentración de sal en pan. Objetivo: Evaluar la aceptabilidad del consumidor del pan del programa piloto, en comparación con un pan sin modificar, en una muestra de panaderías de Santiago. Metodología: Estudio analítico transversal. Selección aleatoria de 5 panaderías piloto y 5 controles. Encuesta a 175 consumidores de cada grupo, para evaluar la aceptabilidad del pan con 5 parámetros sensoriales, con escala hedónica de 5 puntos. Se elaboró un índice de aceptabilidad global ( 5 a 25 puntos), calificando como buena aceptabilidad un valor $\geq 22$ puntos. Resultados: Buena aceptabilidad en todos los parámetros en ambos grupos, con diferencias significativas a favor del grupo control en la calificación del sabor $(p=0,01)$ y aceptabilidad global $(p=0,02)$. El análisis multivariado mostró mejor aceptabilidad en mayores de 60 años $(p=0,01)$ y en panaderías control $(p=0,02)$. Conclusiones: Se encontró mejor aceptabilidad en algunos parámetros del pan no intervenido, aunque la concentración de sodio no fue el único factor que explicó la aceptabilidad.

Palabras clave: sodio, pan, aceptabilidad, hipertensión arterial, Chile.

Agradecimientos: Nuestro más sincero agradecimiento a FECHIPAN por su desinteresada colaboración en la obtención de los permisos para la realización de las encuestas en terreno.

Financiamiento: El financiamiento de este estudio fue realizado por el Departamento de Nutrición de la Facultad de Medicina de la Universidad de Chile en el contexto de desarrollo de una Tesis de Postgrado.

Conflictos de interés: Todos los autores declaran no tener conflictos de interés con las empresas o instituciones vinculadas con el tema estudiado.

\section{BIBLIOGRAFÍA}

1. Umesawa $M$, Iso $H$, Date $C$, Yamamoto $A$, Toyoshima $H$, et al. Relations between dietary sodium and potassium intakes and mortality from cardiovascular disease: the Japan Collaborative Cohort Study for Evaluation of Cancer Risks. Am J Clin Nutr.2008; 88(1):195-202.

2. Meneton $P$, Jeunemaitre $X$, de Wardener $H E$, MacGregor $G A$. Links between dietary salt intake, renal salt handling, blood pressure, and cardiovascular diseases. Physiol Rev. 2005; 85: 679-715.

3. Consensus Action on Salt and Health. Disponible en: http:// www.actiononsalt.org.uk/about/index.html

4. El consumo de sal en Europa. Consejo Europeo de In- formación sobre la Alimentación (EUFIC). Disponible en:http://www.eufic.org/article/es/artid/El-consumo-desal-en-Europal

5. He FJ, Jenner $\mathrm{KH}$, Macgregor GA. WASH-world action on salt and health. Kidney Int. 2010; 78: 745-53.

6. World Action on Salt and Health. Disponible en:http:// www.worldactiononsalt.com/

7. World Action on Salt and Health. Aims. Disponible en: http://www.worldactiononsalt.com/home/aims.htm

8. World Action on Salt and Health.World Salt Awareness Week. Disponible en: http://www.worldactiononsalt.com/ awarenessweek/index.htm/

9. Blanco-Metzler A, Legetic B, Campbell N. Editorial. Arch Latinoam Nutr 2010; (60): 5-6.

10. Estrategia nacional para la reducción del consumo de sal en Chile. Disponible en: http://new.paho.org/hq/dmdocuments/2010/4.\%20EsEscobar\%20Estrategia\%20 Nacional\%20para\%20Reducir\%20el\%20consumo\%20 de\%20Sal\%20Chile.pdf

11. Protección de Salud. Estrategia de Reducción Sal/Sodio en los Alimentos. Ministerio de Salud de Chile. Disponible en: http://www.redsalud.gov.cl/portal/url/page/minsalcl/g_ proteccion/g_alimentos/reduccion_sodio.htm/

12. Technologies of salt reduction in bread: issues, problems and solutions. Seminar: Salt in Bread 21. Oct., 2009, Brussels. Disponible en: http://ec.europa.eu/health/nutrition_physical_activity/docs/ev20091021_salovaara_en.pdf

13. Primo-Yúfera E. Química de los Alimentos. Editorial Síntesis. España 1998. pp 81-95.

14. Lynch E.J, Dal Bello F, Sheehan E.M, Cashman K.D, Arendt E.K. Fundamental studies on the reduction of salt on dough and bread characteristics. Food Rest Int. 2009; 42:885-91.

15. Ni Mhurchu C, Capelin C, Dunford EK, Webster JL, Neal $B C$, Jebb SA. Sodium content of processed foods in the United Kingdom: analysis of 44.000 foods purchased by 21.000 households. Am J Clin Nutr. 2011; 93(3):594-600.

16. Rodgers $A, N e a l B$. Less salt does not necessarily mean less taste. Lancet. 1999; 353(9161):1332.

17. Belz MC, Ryan LA, Arendt EK. The impact of salt reduction in bread: a review. Crit Rev Food Sci Nutr. 2012; 52(6):514-24.

18. Girgis S, Neal B, Prescott J, Prendergast J, Dumbrell S, Turner $C$, Woodward M. A one-quarter reduction in the salt content of bread can be made without detection. Eur J Clin Nutr. 2003; 57(4): 616-20.

19. Bolhuis DP, Temme EH, Koeman FT, Noort MW, Kremer $S$, Janssen AM. A salt reduction of $50 \%$ in bread does not decrease bread consumption or increase sodium intake by the choice of sandwich fillings. J Nutr. 2011; 141(12): 2249-55.

20. Reducción de sal en el pan. Disponible en http://www. indupan.cl/sal.html.

21. Valenzuela K, Vilma Q, Villanueva B, Zavala F, Atalah E. Rev Chil Nutr. 2013; 40(2):119-22. 